\title{
Auditory hallucinations in schizophrenia: the role of cognitive, brain structural and genetic disturbances in the left temporal lobe
}

\author{
Kenneth Hugdahl ${ }^{1,2, *}$, Else-Marie Løberg ${ }^{2}$, Karsten Specht ${ }^{1,3}$, Vidar M. Steen ${ }^{4,5}$, \\ Heidi van Wageningen ${ }^{1}$ and Hugo A. Jørgensen ${ }^{2,4}$ \\ 1. Department of Biological and Medical Psychology, University of Bergen, Bergen, Norway \\ 2. Division of Psychiatry, Helse-Bergen HF, Bergen, Norway \\ 3. Department of Clinical Engineering, Haukeland University Hospital, Bergen, Norway \\ 4. Department of Clinical Medicine/Bergen Mental Health Research Center, University of Bergen, Bergen, Norway \\ 5. Center for Medical Genetics and Molecular Medicine, Haukeland University Hospital, Bergen, Norway \\ Edited by: Robert T. Knight, University of California Berkeley, USA \\ Reviewed by: Patrik Vuilleumier, University Medical Center and University Hospital Geneva, Switzerland \\ Anna C. Nobre, University of Oxford, UK
}

\begin{abstract}
In this article we review research in our laboratory on auditory hallucinations using behavioral and MRI measure. The review consists of both previously published and new data that for the first time is presented together in a cohesive way. Auditory hallucinations are among the most common symptoms in schizophrenia, affecting more than $70 \%$ of the patients. We here advance the hypothesis that auditory hallucinations are internally generated speech perceptions that are lateralized to the left temporal lobe, in the peri-Sylvian region. From this we predict that hallucinating patients should have problems identifying a simultaneously presented external speech sound, as measured through performance on the dichotic listening (DL) paradigm with consonant-vowel syllables, since this technique lateralizes the stimulus input. Across a series of behavioral experiments, we have shown that patients with schizophrenia who experience frequent auditory hallucinations fail to demonstrate an expected right ear advantage on the dichotic listening test. Absence of a right ear advantage is indicative of a functional deficit in the left peri-Sylvian region. The results also revealed that patients with ongoing auditory hallucinations were more impaired than patients with previous hallucinations, and that a higher score on the hallucination item in a standard symptom rating scale (BPRS) correlated negatively with number of correct reports for the right ear stimulus. Moreover, we have found that schizophrenia patients fail to shift attention to the left ear stimulus, when explicitly instructed to focus on the right or left ear stimulus only, thus showing a deficit in inhibition of attention and response-inhibition. The behavioral DL data are substantiated in two MR morphometry studies that revealed significant reductions in grey matter density in the left peri-Sylvian region in hallucinating patients, and patients with reduced left temporal lobe grey matter density. Hallucinating patients also failed to show a right ear advantage in the dichotic listening test. Ongoing fMRI studies are focused on the underlying synaptic and molecular mechanisms by investigating the effects of the glutamate antagonist drug memantine on auditory perception and speech lateralization, and examination of temporal cortex-specific gene expression in the left peri-Sylvian region.
\end{abstract}

Keywords: auditory hallucinations, schizophrenia, hemisphere asymmetry, dichotic listening, memantine, fMRI, VBM

\section{INTRODUCTION}

In this article we summarize empirical work related to auditory hallucinations ${ }^{1}$ in patients with schizophrenia conducted in our laboratory over the last 15 years. The aim of the article is to present the results in a common frame of reference so that it hopefully "tells a coherent story". For this purpose we present data from four behavioral studies using a dichotic listening (DL) paradigm to assess impairment of lateralized speech sound processing in hallucinating and non-hallucinating patients compared

*Correspondence: Kenneth Hugdahl, Department of Biological and Medical Psychology/ Bergen Mental Health Research Center, University of Bergen, Jonas Lies vei 91, N-5009 Bergen, Norway. e-mail: hugdah@@sybp.uib.no

Received: 16 September 2007; paper pending published: 29 November 2007; accepted: 03 January 2008; published online: 28 March 2008.

Citation: Front. Hum. Neurosci. (2008) 1: 6. doi: 10.3389/neuro.09.006.2007

Copyright: @ 2008 Hugdahl, Løberg, Specht, Steen, Wageningen and Jørgensen. This is an open-access article subject to an exclusive license agreement between the authors and the Frontiers Research Foundation, which permits unrestricted use, distribution, and reproduction in any medium, provided the original authors and source are credited. with healthy controls, and structural and functional brain imaging data from two MRI studies. We finally suggest a model for the understanding of auditory hallucinations as instances of perceptual phenomena, possibly related to deficient glutamate regulation, and with a genetic origin. In Study I we have re-analyzed the data presented in Green et al. (1994) on hallucinating and non-hallucinating patients, adding a new healthy control group. In Study II we review data presented in Løberg et al. (2004) examining whether or not ongoing auditory hallucinations have more detrimental effects on dichotic listening performance than having had a previous history of auditory hallucinations. In Study III we present new data on the relationship between frequency of hallucination and dichotic listening performance. In Study IV we review data presented in Løberg et al. (1999) assessing the ability to explicitly focus attention on either the left or right ear stimulus in the dichotic listening situation in hallucinating patients, with the hypothesis that this ability would be impaired in the

${ }^{1}$ Auditory hallucinations can also involve hearing non-verbal sounds, like noise, whistles, etc. We have however restricted our studies to auditory verbal hallucinations, i.e., "hearing voices". 
patients. In the first MRI study we present morphometry (VBM) data on grey matter density in patients and healthy controls, as well as an analysis of cortical thickness. In the second MR study, we present new data from a pharmacological fMRI study where we investigated the effects of the drug memantine, which is a glutamate antagonist, on brain activation when subjects were exposed to dichotically presented speech stimuli.

Hallucinations are one of the most salient positive symptoms in schizophrenia, particularly auditory hallucinations (i.e., "hearing voices") that affects more than $70 \%$ of the patients. In the present context auditory hallucinations are considered perceptual experiences that occur in the absence of a triggering external sensory stimulus (cf. Woodruff, 2004). What characterizes auditory hallucinations is that the patient experiences "someone speaking to him/her" that is perceived as a reality, with an origin outside of the self. For the patient, auditory hallucinations are therefore real perceptions, not imaginations. Such a view would be consistent with an understanding of auditory hallucinations as "derived from inner speech that has been misidentified as coming from outside the self, because of defective self-monitoring" (Frith, 1992). Others have suggested similar views, stating that auditory hallucinations should be regarded as "misrepresentations of inner experiences" (Heinks-Maldonado et al., 2007); "misattribution of speech" (Allen et al., 2007); "misattributed cognition and inner speech" (Kinderman, 2007), and "competition between auditory stimuli and physiological resources" (Hubl et al., 2007). A recent study by Tritsch et al. (2007) points to a possible internal generator for hallucinatory perceptions, possibly caused by developmental neuronal pathology. Tritsch et al. (2007) found that supporting cells in the developing cochlea in the rat brain spontaneously release ATP which initiates bursts of electrical activity in primary auditory neurons before the onset of hearing in newborn rats. Such spontaneous ATP-mediated purinergic P2 receptor-dependent signals rapidly cease after hearing is developed. Supporting cells and hair cells in the mature cochlea continue to express P2 receptors. It is therefore possible that sound-independent activity in the auditory nerve could resume under certain conditions also in adults, through abnormality during ontogenesis, which could lead to auditory hallucinations in patients with schizophrenia.

There are several arguments as to why auditory hallucinations could be regarded as perceptions. The most important argument comes from recent structural and functional neuroimaging studies showing abnormality in the speech perception areas in the peri-Sylvian region in the left temporal lobe (Dierks et al., 1999; Kubicki et al., 2002; Neckelmann et al., 2006). Findings of morphometric changes also go together with altered neuronal activation during hallucinatory episodes, measured with fMRI or PET (Hubl et al., 2007; Shergill et al., 2000, Silbersweig et al., 1995; Woodruff et al., 1997). Since auditory hallucinations occur in the context of language (see e.g., David, 1999; van der Gaag, 2006) it is reasonable to conceptualize such experiences as perceptual phenomena related to speech sound perception, originating in the left hemisphere. This would also be in line with the suggestion by Crow (2004) that the core deficit in schizophrenia is related to abnormal language lateralization, particularly involving the left hemisphere (see also Crow, 1997). Left hemisphere speech perception dysfunction has also been demonstrated in schizophrenia (e.g., Hoffman and McGlashan, 2006). However, if auditory hallucinations are internally generated speech sound perceptions, in the absence of an external acoustic source, why is this experience not suppressed as an "illusion", bur rather is sensed as a real perceptual phenomenon? A possibility is that once initiated, there is a lack of impulse control, or response inhibition, due to failure of what Frith (1992) called defective self-monitoring, that would in addition involve top-down processing strategies localized to prefrontal and parietal brain regions (Fan et al., 2005). Thus, an understanding of auditory hallucinations would need to address both abnormal perception and impaired impulse control. In the review of the empirical work below, we start, however, with describing the bottom-up effects of auditory hallucinations before proceeding to describing the effects of attention modulation as a top-down effect.

\section{Speech perception and hemisphere asymmetry}

An empirical test of whether auditory hallucinations are distortions of speech perception, and lateralized to the left hemisphere, would ideally require an experimental approach assessing both auditory perceptions and lateralization within the same experimental paradigm. The paradigm should, moreover, not be difficult to understand for the patient, and should not involve complex processing strategies and/or response options that would load on other cognitive processes which would confound the perceptual measures. The dichotic listening (DL) paradigm (Bryden, 1988; Hugdahl, 2003; Kimura, 1967) with verbal stimuli, like consonant-vowel (CV) syllables, would fulfil the criteria of both assessing perception, and localization the processing to the left or right hemisphere. Thus, in the behavioral studies described below, we have used a DL paradigm with pair-wise presentations of simple CV-syllables (see Hugdahl and Andersson, 1986 for a detailed description of the paradigm). Research on functional hemisphere asymmetry has shown that the left hemisphere, and particularly the upper and middle parts of the left temporal lobe (peri-Sylvian region), are critical for speech perception and decoding of phonology (Binder et al., 1996; Binder and Price, 2001).

In the Bergen DL paradigm (Hugdahl, 2003; Tervaniemi and Hugdahl, 2003), the subject is presented with two consonant-vowel (CV) syllables simultaneously, one in each ear, and required to report the syllable identified best on each trial. The instruction emphasizes that the subject should answer with only the syllable identified best on each trial in order to tap an initial perceptual process and to avoid confounding with memory since when required to give two answers the subject has to keep one syllable in working memory while answering with the syllable initially perceived. Moreover, the subject is not told that there are two different syllables on each trial, and also encouraged "not to try to remember, or think about the stimulus, but just report what you initially hear". Hearing threshold differences between the ears are typically tested with audiometer screening procedures (or similar procedures). The DL stimuli consist of the six stopconsonants $/ \mathrm{b} /, / \mathrm{d} /, / \mathrm{g} /, / \mathrm{p} /, / \mathrm{t} /$, and $/ \mathrm{k} /$ paired with the vowel $/ \mathrm{a} /$, to form the six CV-syllables $/ \mathrm{ba} /, / \mathrm{da} /, / \mathrm{ga} /, / \mathrm{pa} /, / \mathrm{ta} /, / \mathrm{ka} /$. A single run consists of 36 trials including all possible pair-wise combinations of the six syllables. The six homonymic trials are, however, excluded from statistical analysis since they represent the same stimulus in both ears. Thus, the number of analyzed trials in a run is 30 .

The basic feature of the dichotic situation is to provide more elements to be processed at any point in time than the brain is capable of The question then becomes which element or component of the stimulus configuration input will be selected. The typical result is that more correct items are reported from the right ear, also when controlling for threshold differences in hearing between ears (see Hugdahl, 2003). This is labelled a right ear advantage (REA) and is a quite robust marker of left hemisphere speech perception lateralization, replicated in hundreds of studies since first introduced by Kimura (1961). ${ }^{2}$ The DL test, or paradigm, is sometimes repeated three times with different instructions to attend to and report only from the right or left ear stimulus, or no instruction of attention focus (see Hugdahl and Andersson, 1986). By instructing the patients to focus attention and respond to only the right or left ear stimulus, it is possible to assess the contribution of attention in addition to stimulus perception. In addition, instructing the patient to focus attention on the left ear stimulus produces a top-down/bottom-up processing conflict since the stimulus-driven bottom-up effect pushes for selecting the right ear stimulus, while the instruction-driven top-down effect pushes for selecting the left ear stimulus.

A frequent explanation for the REA is the neuroanatomical model suggested by Kimura (1967) which states that the contralateral auditory neural pathway is more preponderant than the ipsilateral one (Brodal, 1981), and that the left hemisphere is dominant for speech perception

${ }^{2}$ Kimura (1961) is correctly credited for introducing the dichotic listening technique to neuropsychology, although she did not use a CV-syllables paradigm. 


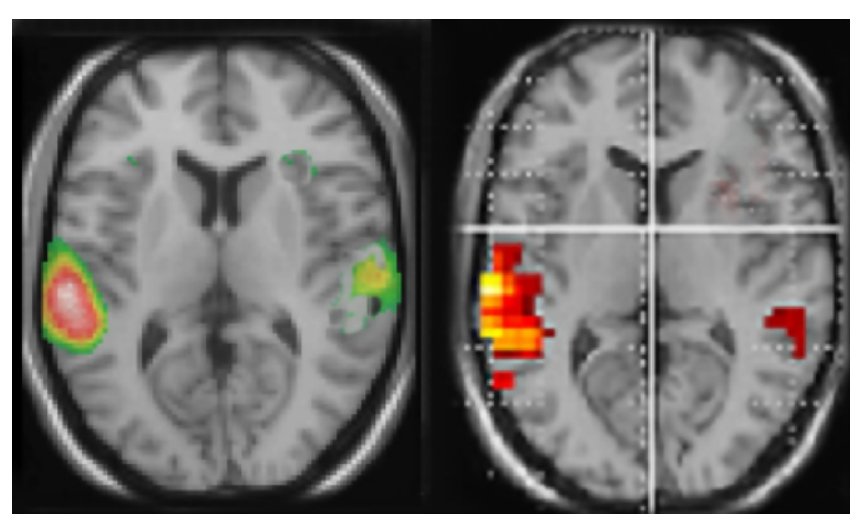

Figure 1. Functional brain activation data when healthy subjects listen to dichotic presentations of consonant-vowel syllables. Note the marked left hemisphere lateralization in both data sets. The left-hand panel shows PET-data (from Hugdahl et al., 1999), the right-hand panel shows corresponding fMRI-data (unpublished data Bergen fMRI-group). Both data sets were evaluated with a statistical significance threshold at $p<0.001$ (uncorrected). Neurological left-right display conventions.

(Sperry, 1974). This should favor processing of the right ear stimulus because it would have direct access through the contralateral auditory pathways to the speech processing areas in the left temporal lobe. A recent brain lesion study by Pollmann et al. (2004) also showed that the left ear stimulus needs to be transferred across the corpus callosum from the right to the left hemisphere in order to be processed, which is additional support for the Kimura neuroanatomical model.

Neuroimaging research has shown differential blood flow distribution to the left and right superior temporal gyri during dichotic presentations of CV-syllables (Hugdahl et al., 1999), demonstrating asymmetrical activation for processing of dichotically presented CV-syllables in the left and right hemispheres. Figure 1 shows ${ }^{15} 0$-PET and fMRI-data in healthy subjects when processing dichotically presented CV-syllables, revealing a left-sided lateralization in the peri-Sylvian region. In both the PET and fMRI studies there was also a significant $(p<0.05)$ REA (i.e., significantly more correct items reported for the right ear stimulus, corroborating the brain imaging results).

\section{DICHOTIC LISTENING BEHAVIORAL RESULTS}

The Norwegian studies reviewed below were approved by the Regional Committee for Medical Research Ethics in Western Norway, and subjects were treated in accordance with the Helsinki Declaration.

\section{Study I}

Study I involved 45 patients from the inpatient units of Camarillo State Hospital in California (originally published in Green et al., 1994), with 21 non-hallucinating and 24 hallucinating patients. The data in the Green et al. (1994) study were re-analyzed, and compared with a new group of 50 healthy control subjects randomly drawn from the Bergen $\mathrm{DL}$ database.

The patients underwent a diagnostic interview based on the Structured Clinical Interview for DSM-III-R (Spitzer et al., 1990) and the psychotic symptom sections of the Present State Examination (Wing et al., 1974). Potential subjects were excluded if they had an identifiable neurological disorder, or signs of mental retardation. No subjects were excluded for these reasons. All patients were on typical antipsychothic medication, with a few patients having a history of substance dependence, although none had abused substances in the last months prior to testing.

The hallucinating and non-hallucinating groups were comparable in gender distribution, age, education, medication, time since first institu-
Table 1. Demographic data for the hallucinating and non-hallucinating patients.

\begin{tabular}{lcc}
\hline & $\begin{array}{c}\text { Hallucinating } \\
\text { pat }\end{array}$ & $\begin{array}{c}\text { Non-hallucinating } \\
\text { pat }\end{array}$ \\
\hline Age & 35.2 years & 36.1 years \\
Education & 11.7 years & 11.7 years \\
Duration of & 12.16 years & 16.17 years \\
Illness & & \\
Positive BPRS Symptoms & 4.39 & 3.48 \\
Negative BPRS Symptoms & 8.65 & 8.09 \\
Medication (Chlorpromazine & 1347 & 1182 \\
Equivalents) & & \\
\hline
\end{tabular}

tionalization, and verbal IQ. Demographics are shown in Table 1. None of the comparisons between the groups in Table 1 were significant.

The majority of the patients were right-handers with one left-handed patient in the non-hallucinating group and six patients in the hallucinating group. The patients received an expanded version of the Brief Psychiatric Rating Scale (BPRS) within two weeks of testing. It is true that the BPRS interview does not uniquely assess auditory hallucinations (Lukoff et al., 1986). Thus, findings could have been confounded by some patients experiencing visual or other sensory modality hallucinations. Although this can not completely be ruled out, the clear majority of hallucinations in schizophrenia are auditory in nature, and perhaps more importantly, it is not likely to get a score in the upper range (equal to or larger than "4") on the BPRS hallucination item if the experience does not involve hearing voices. It would also be difficult to explain why a visual or tactile hallucinatory experience should interfere with performance on an auditory task, like $\mathrm{DL}$.

The patient groups had equal scores on conceptual disorganization (formal thought disorder) and on the withdrawal/retardation index (blunted affect, emotional withdrawal, and motor retardation). The hallucinating group had higher scores on unusual thought content, which indicates a higher degree of delusional thinking, although the means for both groups were in the psychotic range. At the beginning and at the end of the testing session, all subjects were administered a brief interview to determine the time of the most recent hallucinatory experience. In addition to the BPRS questionnaire scores, the patients were asked just before the study to (1) give verbatim examples of recent auditory hallucinations, (2) indicate that the perceptual experience had an auditory quality as opposed to a thought quality, and (3) indicate that it occurred in a clear state of consciousness. Of the patients in the hallucinating group, $75 \%$ clearly reported auditory hallucinations during the testing session. Three patients reported the presence of auditory hallucinations during one hour before testing, while three patients reported the presence of auditory hallucinations during the two hours before testing. If patients denied at both the beginning and the end of testing that any auditory hallucinations had occurred that day, they were included in the non-hallucinating group.

The data were analyzed in a factorial ANOVA with groups and ear as factors, and the results are seen in Figure 2. The results showed that the non-hallucinating group performed equally well as the healthy control subjects with regard to the magnitude of the REA, although with significantly lower overall performance $(p<0.05)$. The control and nonhallucinating groups both differed from the hallucinating group with regard to the right ear advantage, as indicated by a significant two-way interaction of Groups $\times$ Ear $(p<0.05)$. In order to control for the effect of handedness on the DL scores (since handedness has been shown to relate to hemispheric asymmetry) the data were analyzed with and without the left-handed subjects. There were six left-handers in the hallucinating group and one left-hander in the non-hallucinating group. Excluding the left-handers did not significantly affect the results. When 


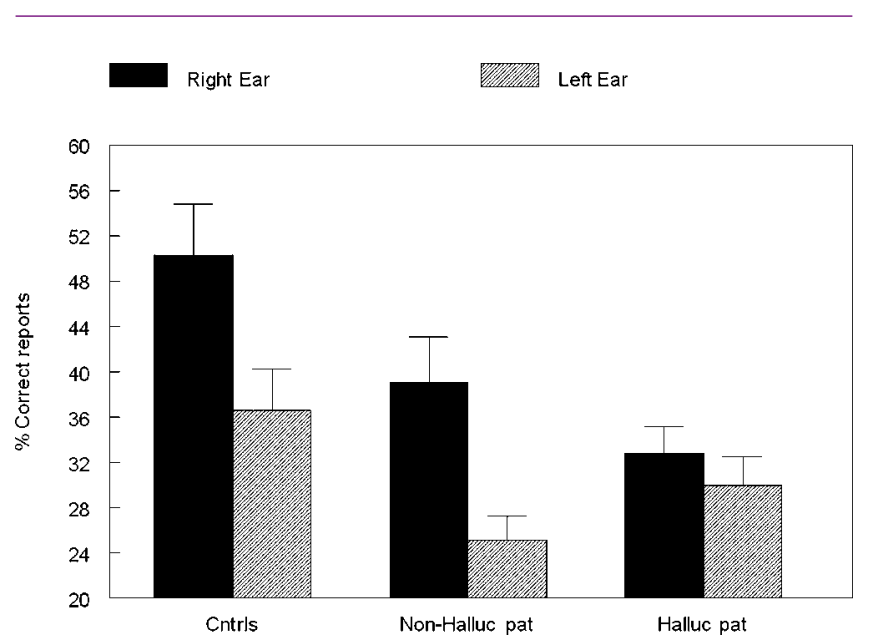

Figure 2. Percent correct reports on the CV-syllables DL paradigm split for right and left ear stimulus presentations, and healthy controls, nonhallucinating and hallucinating patients with schizophrenia.

looking at the distribution of individual scores from the right and left ear, the non-hallucinating patients showed a distribution with 16 of $21(76 \%)$ with a REA, which would be expected from the healthy control group data. The hallucinating patients were scattered more evenly, with only 12 of the $24(50 \%)$ patients showing a REA. The finding that patients with auditory hallucinations did not show the normal REA when tested with the dichotic presentations of simple CV-syllables was surprising and set the stage for further hypotheses regarding the neurocognitive origin of hallucinations. The findings reviewed in Study I have a further implication in that the results point to a perceptual basis for hallucinations since failure of processing of the dichotic CV-syllables is a perceptual failure.

\section{Study II}

In the second study we review data presented in Løberg et al. (2004). We asked the question whether or not the findings in Study I were primarily caused by state or trait effects, i.e., by ongoing or previous hallucinations. We therefore investigated $\mathrm{DL}$ performance in a heterogeneous group of 26 patients who were compared with 18 healthy control subjects. The patients were recruited from diverse treatment facilities in the Bergen area that included both in- and outpatient clinics, with both acute and chronic treatment units. All patients met ICD-10 (World Health Organization, 1992) and DSM-IV and research criteria for schizophrenia (American Psychiatric Association, 1994), diagnosed by means of the Structural Clinical Interview for DSM-IV (SCID) by an experienced psychiatrist. The Positive and Negative Symptoms Scale (SCI-PANSS) was used for symptom ratings (Kay et al., 1989). All patients received medication, with median haloperidol-equivalents per day of $10 \mathrm{mg}$. The patients and healthy control subjects underwent the same DL protocol as the patients in the Green et al. (1994) study. The DL results are shown in Figure 3.

The patient group was further split into two sub-groups, with one sub-group having a PANSS score of "4" or higher which would indicate ongoing hallucinations, and another sub-group with a PANSS score of "2" or less, but with a history of hallucinations, and with two patients not showing any signs of hallucinations. Previous experience with hallucinations was also recorded through a specially designed questionnaire, and through the patients' records. The auditory quality of the hallucinations was validated through an interview questionnaire used by the therapist, specifically designed for this study, in addition to the patient's records and the SCID interviews.

The results showed a significant overall difference between the patients and the healthy controls, with fewer correct reports in the patient

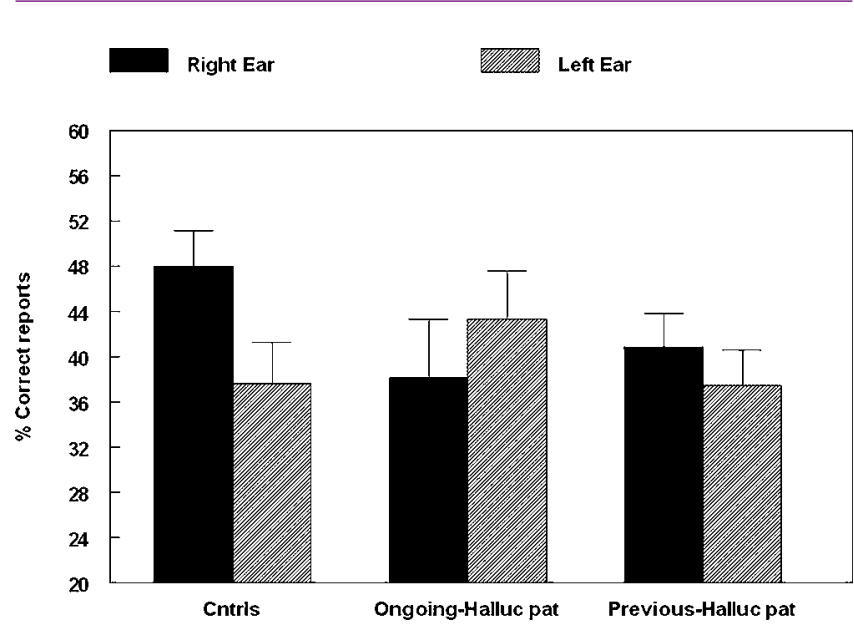

Figure 3. Percent correct reports on the CV-syllables DL paradigm split for healthy controls, patients with ongoing and patients with previous auditory hallucinations.

group $(p<0.05)$. The ongoing hallucinations group, moreover, failed to show a REA, and was significantly different from both the healthy control group $(p<0.05)$ and the previous hallucination group $(p<0.05)$, as indicated by the significant two-way interaction of Group by Ear. Thus, there was a marked difference in performance on the DL paradigm for patients with ongoing hallucinations vs. patients with previous hallucinations. The reduced REA in the ongoing hallucinations sub-group indicates that state factors may be more important when it comes to the ability to process an external speech sound presented to the left hemisphere in hallucinating patients.

\section{Study III}

In the third study we present new data ${ }^{3}$ related to the hypothesis that if auditory hallucinations are speech mis-perceptions, then the reduction of the REA would be greater for patients experiencing more frequent hallucinations than for patients experiencing few or no hallucinations. We therefore compared a group of patients scoring a "1" (no hallucinations) with a group scoring a "4" or higher (frequent hallucinations) on the BPRS hallucination item, and then compared performance on the DL paradigm in the two sub-groups. There were 37 patients with "no hallucinations" and 44 patients with "frequent hallucinations". The two patient groups were also compared with a group of 50 healthy control subjects. The means for the three groups are seen in Figure 4.

An ANOVA showed a significant interaction between Groups and Ear $(p<0.005)$, and follow-up tests showed that the interaction was due to failure of a significant REA in the "frequent hallucinations" patient group, while the "no hallucinations" patient group and the healthy control group both had a significant REA.

We also had the opportunity to correlate the DL scores with positive and negative symptoms using the entire range of BPRS questionnaire scores from "1" to "7". The DL right and left ear scores were converted to a laterality index score defined by the formula: [(right ear score - left ear score)/(right ear + left ear score) $] \times 100$. This analysis was done on an extended sample of 101 patients diagnosed with schizophrenia. The objective was to rule out that the relationship between $\mathrm{DL}$ performance and hallucinations described in Studies I-III above would replicate for all symptoms in the BPRS questionnaire, thus suggesting that the results would be more related to overall illness severity rather

${ }^{3}$ The patients were an extended sample of patients from Bergen Clinics and from the former Camarillo State Hospital, CA, USA not previously reported for the whole sample. Some of the patients were included in Løberg et al., 1999, and Hugdahl et al., 2008. 


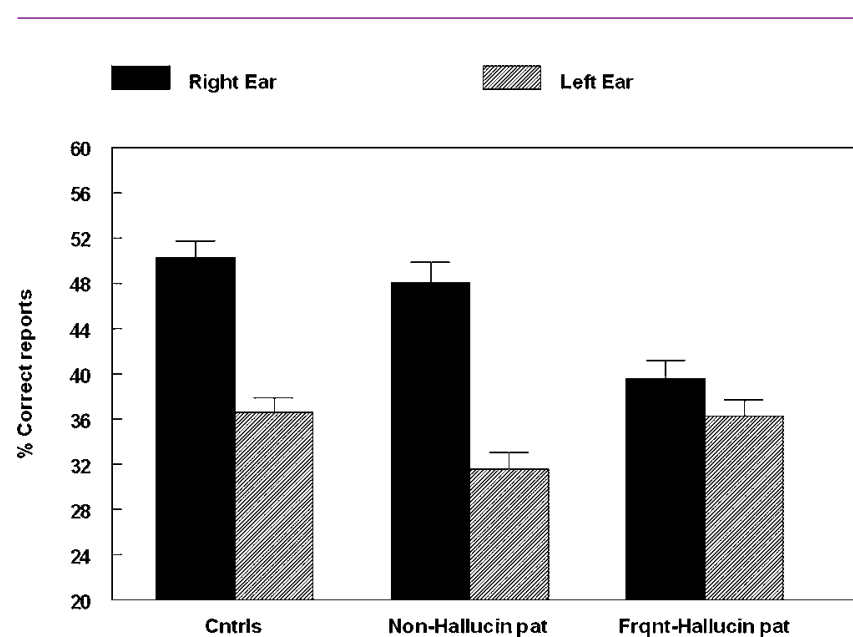

Figure 4. Percent correct reports on the CV-syllables DL paradigm split for right and left ear stimulus presentations, and healthy controls, nonhallucinating and frequently hallucinating patients.

than symptom specificity. The results showed a significant correlation only for the positive BPRS symptoms $(r=-0.31, p<0.05)$, while total BPRS scores $(r=-0.13$, n.s.) and negative symptoms $(r=-0.03$ n.s.) showed non-significant correlations with DL performance. The positive symptoms in the BPRS questionnaire include items related to delusions and conceptual disorganization in addition to hallucinations. The correlation analysis revealed that delusions and conceptual disorganization are related to impaired performance on the $\mathrm{DL}$ test. Because the thematic content of auditory hallucinations is often delusional and conceptually disorganized, it could therefore be predicted that these items should be correlated. It is probably rare to find a patient with frequent and/or intense auditory hallucinations who not also shows frequent signs of delusions, and consequently would get a high score on the BPRS unusual thought content item. Since there was no significant correlation between DL performance and the total BPRS score we can rule out that the reported significant effect for hallucinations was caused by illness severity in general.

\section{Study IV}

In the Løberg et al. (1999) study we addressed the issue whether selfmonitoring and response inhibition may also be impaired in patients with schizophrenia, and should be factors to consider when trying to understand the neurocognition of auditory hallucinations. Moreover, an understanding of the neurocognitive basis for auditory hallucinations should take into account the ability to shift attention away from the voices. This would engage other cortical areas and networks, particularly in fronto-parietal regions (Posner and Raichle, 1994; Posner and Rothbart, 2007). Hallucinations involve a shift of attention towards the "voice", when the voices are kept active in working memory, while successful therapy may involve getting the patient to orient attention away from the "voices".

From this follows a prediction that attention to an external auditory source, like CV-syllables, would also be attenuated in patients with auditory hallucinations. Since attention activates a fronto-parietal network, attention failure in the forced-attention DL task, in hallucinating patients, could be linked to the well-known hypofrontality syndrome (Blakemore and Frith, 2000) in schizophrenia, in general. By instructing the patients to actively shadow only the right, or left, ear stimulus when undergoing the DL paradigm (Hugdahl and Andersson, 1986), this prediction could be empirically tested. Figure 5 show data from Løberg et al. (1999) based on 33 schizophrenia inpatients and 33 healthy control subjects. The mean BPRS score on the hallucination item was 4.85 (SD 2.29),

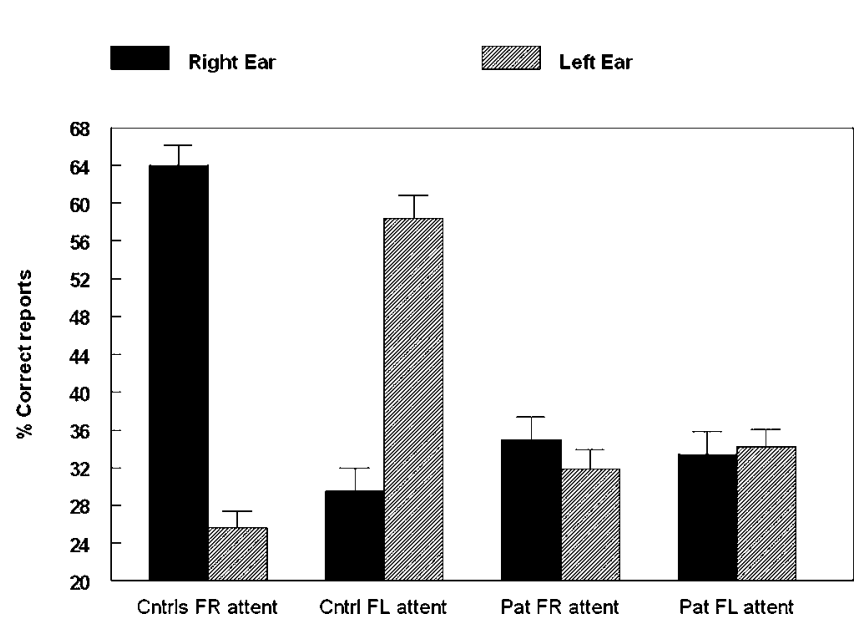

Figure 5. Percent correct reports on the CV-syllables DL paradigm split for right and left ear stimulus presentations, and healthy controls and patients, when the subjects were instructed to focus attention on either the right ("forced-right") or left ("forced-left") ear stimulus.

which indicates that the patients had frequent hallucinations. The DL results showed that the patients were significantly $(p<0.05)$ impaired compared to the healthy control subjects during the forced attention conditions. Thus, the patients showed a deficit in the ability to inhibit a stimulus-driven right ear response and shift attention focus to the right or left ear stimulus.

The implication of this is that patients with schizophrenia seem to be impaired in using a self-initiated top-down processing strategy to overcome a stimulus-initiated bottom-up REA, independently of whether they fail to show a stimulus-driven REA in the first place.

\section{SUMMARY AND DISCUSSION}

\section{OF BEHAVIORAL DATA}

To summarize, the $D L$ data showed an inverse relationship between auditory hallucinations and right ear performance, supporting the idea that auditory hallucinations interferes with the perception of an externally presented speech sound, localized to the left temporal lobe. What is not clear from the DL results is whether this is caused by a direct interference at the time of "hearing voices" or that having experienced hearing voices over a long time is a consequence of pathology at the neuronal level localized to the left peri-Sylvian region.

The results from Study I and II may indicate that both factors are operative. In Study I we also compared DL performance for a sub-group of the hallucinating patients when they were both in a hallucinating and non-hallucinating state. The results showed no difference in performance, with close to zero difference between the right and left ear scores in both states. This indicates that having repeated frequent experiences with hallucinations is a predictor of failure of DL performance, which points to a pathology that causes distortions of perception related to speech and language (cf. Allen et al., 2007; Heinks-Maldonado et al., 2007), and/or dysfunction of language-related neurocognitive networks (Plaze et al., 2006). The results in Study IV indicates that hallucinating patients, in addition to showing an abnormal perceptual response, also may have deficient source monitoring (cf. David, 1999; 2004) and an inability to use attention to cognitively suppress or inhibit a perceptually salient stimulus.

\section{MR MORPHOMETRY DATA}

The next main question asked was whether there would be markers of brain tissue abnormality or pathology in the left temporal lobe in patients with schizophrenia, and particularly in patients with frequent auditory 
hallucinations, which would explain the failure of such patients to show an expected REA on the DL task. For example, Kubicki et al. (2002) reported that patients with schizophrenia had significantly reduced grey matter density in the left peri-Sylvian region (see also DeLisi, 1999). Moreover, Silberzweig et al. (1995) reported increased regional blood flow in this region when patients were actively hallucinating (see also Shergill et al., (2000, 2004), and Hoffman et al. (2007) found that applying transcranial magnetic stimulation (TMS) to the left peri-Sylvian region interfered with ongoing auditory hallucinations, thus pointing towards a new way of treatment that would affect both perception, attention, and self-regulation.

With these findings as a backdrop we had the opportunity to scan a sub-sample of the patients participating in the Hugdahl et al. (2004) fMRI-study on working memory deficits in schizophrenia, using voxelbased morphometry (VBM) to quantify differences in grey matter density between the patients and a healthy control group (see also Neckelmann et al., 2006). VBM is a semi-automated parametric MR imaging technique (Ashburner and Friston, 2001) to statistically evaluate differences on a voxel-by-voxel basis between two groups of subjects, in our case, a group of patients with schizophrenia and a healthy control group, for the identification of regionally specific differences in gray or white matter volume concentration without having to focus on a predefined region of interest.

\section{Voxel-based morphometry of grey matter density} and hallucinations

We split the patients $(n=12)$ on whether they scored a " 1 " or " $>1$ " on the BPRS questionnaire hallucination item, thus sub-dividing the sample into non-hallucinating $(n=6)$ and hallucinating $(n=6)$ sub-groups, and then compared the two groups with a group of healthy control subjects $(n=12)$. The objective was to investigate whether patients with schizophrenia who fail to show a REA in the DL test also show signs of brain abnormality in regions related to the left peri-Sylvian region. All subjects were scanned with a Siemens 1.5 Tesla MR scanner. It should be mentioned at the outset though that since the size of the sub-groups was quite small, the VBM results should be read with caution and firm conclusions not be drawn.

The results are seen in Figure $6 \mathrm{~A}$ and showed that hallucinating patients had significantly reduced ( $p<0.001$, uncorrected) grey matter density in the left superior temporal gyrus, the medial prefrontal cortex in peri-ventricular areas, and in the thalamus. The non-hallucinating patients (seen in the right-hand panel) did not show any comparable reductions.

\section{Voxel-based morphometry of grey matter density and REA}

A second analysis involved splitting the patients into those showing a REA $(n=7)$ on the DL task vs. those that showed no ear advantage, or a left
Hallucinating patients
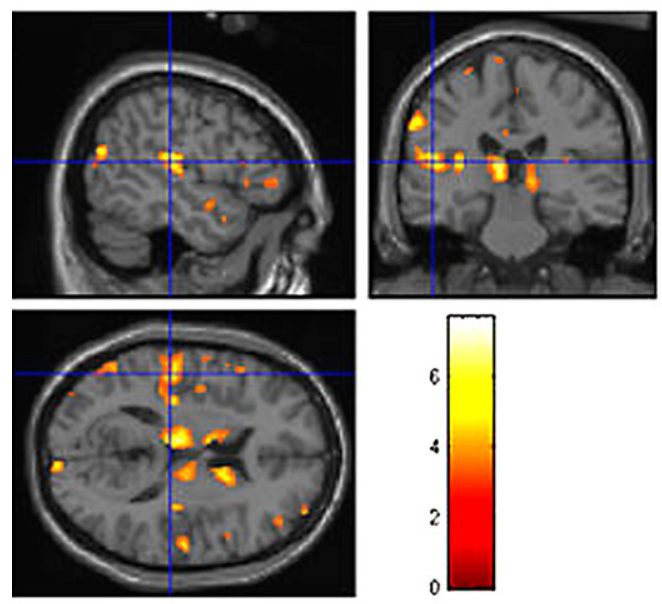

Patients that failed to show a REA
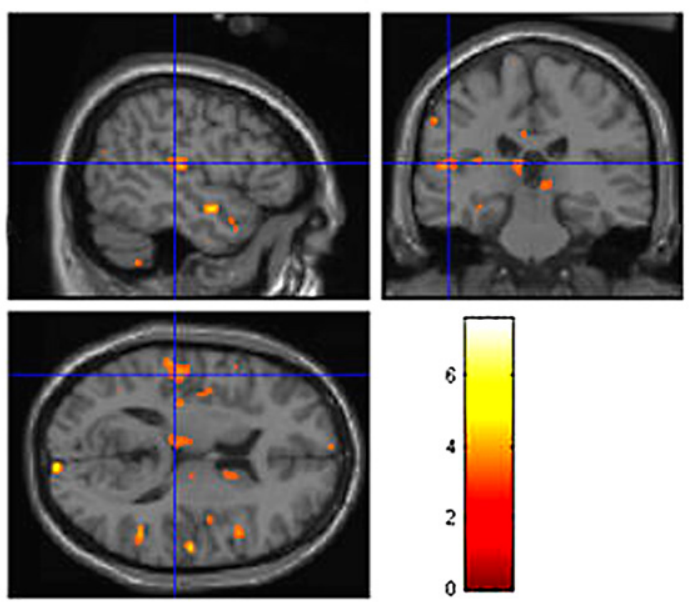

Non-Hallucinating patients
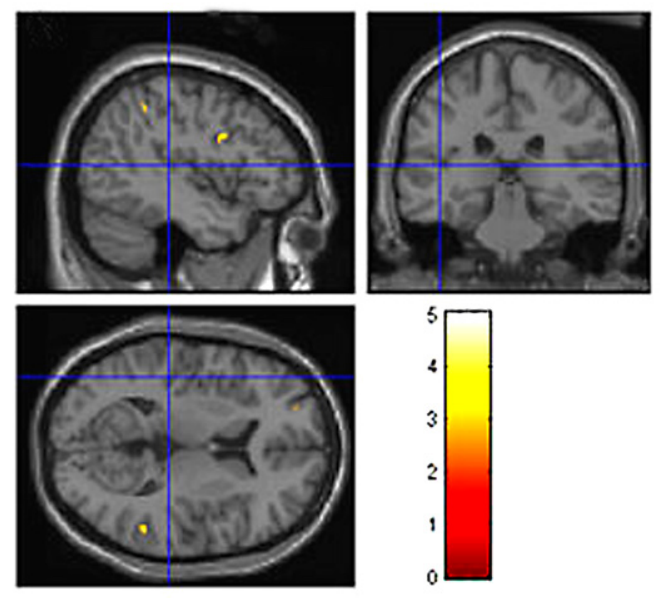

Patients that showed a REA
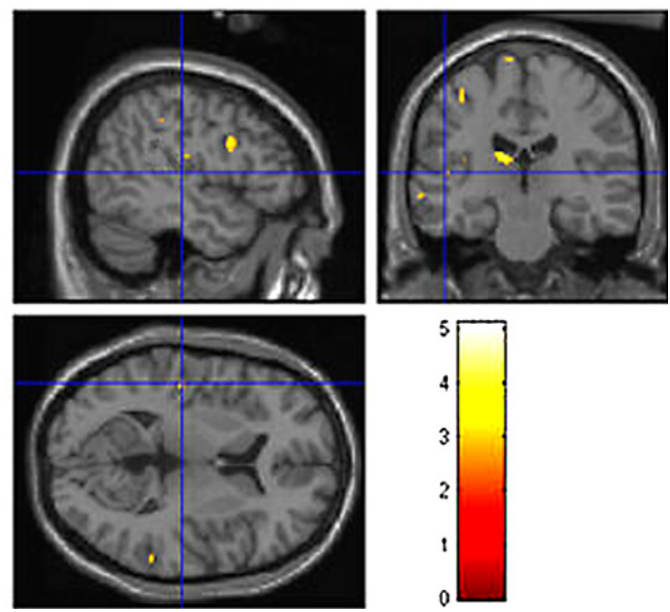

Figure 6. Colored areas show significant reductions in grey matter density in the schizophrenia patients compared to healthy control subjects. The upper panel shows the sub-group of hallucinating (left) and non-hallucinating (right) patients compared to controls. The lower panel shows the sub-groups of patients that failed (left) respectively managed (right) to show a REA on a dichotic listening test. 
ear advantage $(n=5)$ and then applied the VBM analysis for differences in grey matter density between the sub-groups of patients and the same healthy control group $(n=12)$ (see Figure 6B). A REA was defined as having at least one more correct report for the right ear stimulus. A left ear advantage (LEA) was correspondingly defined as having at least one more correct report for the left ear stimulus, and a no ear advantage (NEA) was defined as having an equal number of correct reports for the right and left ear stimulus. The results from the REA VBM analysis are shown in Figure 6B.

The sub-group of patients who failed to show a REA (left-hand panel) had significant $(p<0.001$, uncorrected) reductions in grey matter density, particularly in the left peri-Sylvian and thalamic areas, that was not seen in the patients with a REA on the DL paradigm (right-hand panel). The patients who failed to show a REA also had scattered grey matter reductions in the right hemisphere, while the patients who showed a REA had a small area with grey matter reduction in the left prefrontal cortex. The grey matter reductions in thalamic areas in the hallucinating patients can be interpreted as a gating failure, or deficit. Hugdahl et al. (1990) found that intracranial electric stimulation of thalamic nuclei in Parkinson patients undergoing stereotactic surgery for tremor symptom relief, also affected the ear advantage when they were simultaneously tested on the DL paradigm. They suggested that the thalamus may act as a "gating switch" to gate auditory input to the processing areas in the temporal cortex. We therefore suggest that the results may be interpreted as a gating deficit in schizophrenia (cf. Siegel et al., 1984).

\section{Freesurfer analysis of cortical thickness}

To further pursue a suggested pathology in the speech processing areas of the left temporal lobe in schizophrenia (Dierks et al., 1999; Gaser et al., 2004; Lawrie and Abukmeil, 1998; Neckelmann et al., 2006) we also analyzed differences in thickness of the cortical mantle, using the Freesurfer software, for the same patients that participated in the VBM study. Analysis of cortical thickness would reflect possible additional grey matter pathology related to the architecture of the cortical foldings that could be a marker of neuronal displacements, like polymicrogyria and neuronal migration disturbances, which in turn could point to a neurodevelopmental abnormality in schizophrenia, also related to hallucinations.

Cortical thickness was estimated as the distance between the gray/ white boundary and the outer cortical surface, resulting in a continuous estimate across the cortical mantle. Regional cortical thinning has been demonstrated in aging and related clinical states such as dementia (Espeseth et al., 2006; Salat et al., 2004), that also has been shown to correlate with functional outcomes including performance on cognitive tests, with reduced thickness and tissue volume associated with decreased cognitive capacity (Fjell et al., 2006). Although the reasons for the functional correlations with cortical thinning are not entirely known, it has been suggested that number of neurons or synaptic connections, dendritic expansion, or myelin thickness are related to the thickness of the cortical mantle (Deary and Caryl, 1997). Figure 7 show the results of the Freesurfer analysis.

Thus, it is reasonable to assume that if patients with schizophrenia and particularly patients with frequent hallucinations, show reductions in grey matter density compared to healthy controls, corresponding to a failure to show an expected REA in the DL task, then it is reasonable to assume that they would also show signs of cortical thinning.

Figure 7 shows that the most pronounced difference in cortical thickness between the patients and the control subjects was in the left temporal lobe area in the peri-Sylvian and planum temporale areas, indicated by the "boomerang-shaped" area in Figure 7. Taken together, the MR morphometry results indicate that patients with schizophrenia show pathology at the neuronal level in the speech perception areas in the left hemisphere.

\section{BOLD fMRI and REA}

The MR morphometry results were followed-up in a recent fMRI study in our laboratory, where 14 patients with a diagnosis of schizophrenia and

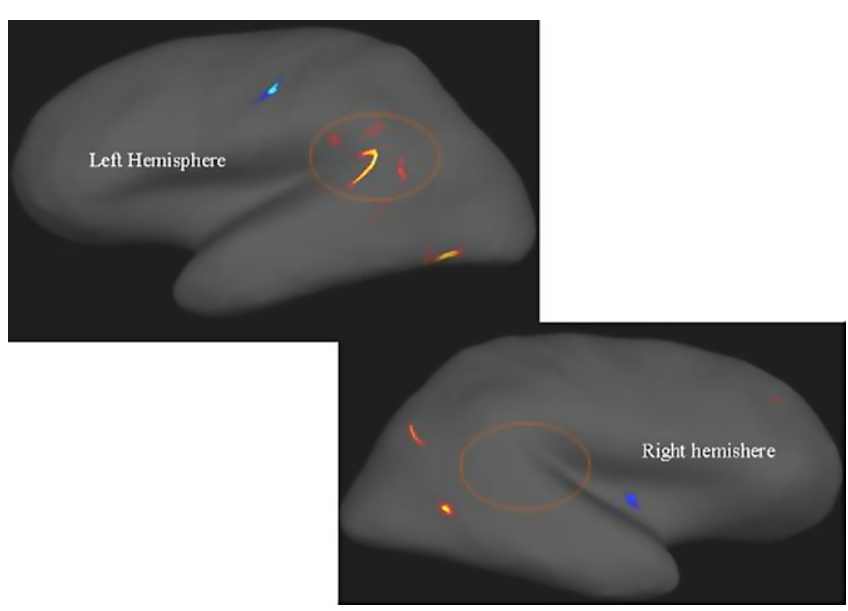

Figure 7. Areas with warm (red, yellow) colors show areas with reduced cortical thickness in patients with schizophrenia compared with healthy controls. Note the marked asymmetry in the peri-Sylvian region inside the circles. Areas in cold colors (blue) show increased thickness in the patients. Significance threshold set to $p<0.001$ with a cluster extension of at least 10 voxels. Neurological left-right display conventions.

16 healthy controls were scanned while listening to dichotic presentations of the CV-syllables. The subjects were scanned with a GE Signa 3.0 T scanner, which would allow for better spatial resolution, and the data analyzed with the SPM5 software. A $p<0.05$ (corrected) significance threshold revealed significant reduction in activation in the patients in the superior and medial temporal gyri, on both the left and right side, but with a relatively larger reduction on the left side compared to the healthy controls.

The patients in the fMRI were defined as "non-hallucinating" on the PANSS hallucination item. This could explain why we did not see increased activation in the left peri-Sylvian region that has been observed in hallucinating patients (Dierks et al., 1999; Shergill et al., 2000). It therefore remains to be demonstrated whether patients with frequent hallucinations that show reduced grey matter density and fail to show an expected REA on the DL task, show increased or decreased neuronal activation in an fMRI situation. Both outcomes could be predicted, depending on the functional significance of the grey matter abnormality in the left temporal lobe. One possibility is that the functional differences are due to volumetric changes in the brain of hallucinating patients, possibly caused by atrophy. This is however unlikely since unpublished data from our lab have shown that BOLD activation changes do not seem to follow volumetric or perfusion changes in general.

\section{SYNAPTIC MEDIATION AND THE GENETICS OF AUDITORY HALLUCINATIONS}

A series of ongoing studies in our laboratory is addressing the question of mediating mechanisms at the synaptic and genetic levels that may mediate the behavioral and brain structural and functional findings. We are currently working along the lines that a deficit in glutamate function may be instrumental in mediating the failure to show a REA in the DL test. It is known that schizophrenia patients show lower levels of glutamate and we are currently collecting data on glutamate concentrations in the left and right temporal lobe areas, in the peri-Sylvian region using MR spectroscopy, and to correlate the spectroscopy results with frequency of hallucinations and DL performance.

\section{NMDA receptor function}

We recently performed an $\mathrm{fMRI}$ study where the effect of glutamate on cognitive function was investigated by manipulating levels of glutamatergic 
transmission through administration of memantine (Ebixa $\left.{ }^{\circledR}\right)$. Memantine reduces the action of glutamate in the NMDA receptor and is a frequently used drug for treating Alzheimer's disease because it reduces the excitotoxic effect of increased accumulation of glutamate caused by the disease process. Excess glutamate in brain parenchyma has excitotoxic effects. The study involved 28 healthy participants aged 20-30 years who were taking memantine orally during 21 days, gradually increasing the dose from 10 to $20 \mathrm{mg}$ to obtain a steady-state concentration before the subjects were tested with the DL paradigm while in the MR scanner. The design was a cross-over design so that all subjects also participated when not on memantine, thus using a within-subjects control procedure.

The fMRI data were acquired on a 3.0 Tesla GE MR scanner, using a block-design with 30 second alternating ON- and OFF-blocks. We used a sparse sampling image acquisition technique where the CV-syllables were presented in "silent gaps" when the scanner was turned off. This was done in order not to confound the BOLD responses to the CV-syllables stimuli with the BOLD responses to the scanner noise. We also used a new oral response technique where the subjects responded orally after each trial which CV-syllable they had heard, making the fMRI DL paradigm identical to the behavioral DL paradigm described above (van den Noort et al., 2007). The BOLD data were analyzed with the SPM5 analysis software package. The significance threshold was set to $p<0.05$, FWE-corrected for multiple comparisons and with a cluster extension of 10 voxels for the main-effects analysis, reducing the threshold to $p<0.01$ and 0.05 for the between-condition comparisons.

Preliminary results are seen in Figure 8 . The results show that memantine has the effect of reducing neuronal activation in the periSylvian region, particularly on the left side. When comparing the memantine and drug-naïve conditions against each other, there were remaining significant activations for the drug naive condition minus the memantine condition contrast that was not seen when reversing the contrast. Since the NMDA receptor is widely distributed across brain regions, we expected that memantine would have a general effect of reducing brain activation in an extended activation network that would correspond to the overall effect of glutamate in the brain. This was also seen in the fMRI results. The fMRI results were paralleled in the behavioral data with reduction of the magnitude of the REA in the memantine condition.

Thus, although reasoning by analogy from the results on healthy individuals, we suggest that glutamatergic transmission may be deficient in auditory hallucinations which may trigger the experience of hearing voices (cf. Bartha et al., 1999; Tritsch et al., 2007). The analysis of the MR spectroscopy data may further support such a hypothesis that would corroborate the behavioral and fMRI data. We intend to follow-up the memantine study with a corresponding study on hallucinating and nonhallucinating patients with schizophrenia.

As for the genetics of auditory hallucinations, we are currently using microarray technology for asymmetry of gene expression in the left and right temporal lobe, particularly in the peri-Sylvian region. Genetic vulnerability may predispose for auditory hallucinations in patients with schizophrenia. The aim of these studies is to identify new candidate genes that affect speech perception in general, but also would affect susceptibility to develop auditory hallucinations in particular. Speech and language disturbances are likely to display complex inheritance, involving several different genes (Fisher et al., 2003), although some rare monogenic disorders are also known.

As an example, mutations in the forkhead-box transcription factor gene FOXP2 cause severe speech deficits in combination with impairments in expressive and receptive language (Lai et al., 2001; Vargha-Khadem et al., 1995). Patients with abnormal FOXP2 function show disturbed activation of language-related brain regions, such as underactivation of Broca's area (Liegeois et al., 2003). Interestingly, polymorphisms in FOXP2 have recently been associated with auditory hallucinations in schizophrenia (Sanjuan et al., 2006). The cholecystokinin type A receptor (CCK-AR) is another gene that has been suggested to confer susceptibility to auditory hallucinations (Sanjuan et al., 2004; Toirac et al., 2007; Wei and Hemmings, 1999). Due to the recent finding by Sun et al. (2005) of a gene (LM04) being expressed differentially in the left and right peri-Sylvian region in embryonic human brains, it is now an open question whether an abnormality in gene expression in the left vs. right peri-Sylvian area may be a factor behind auditory hallucinations. The finding by Sun et al. (2005) also fits nicely with previous findings that the left hemisphere is more vulnerable for disturbances during ontogenesis (Gogtay et al., 2004).

We have recently shown that gene expression profiles in the rat brain disclose regional patterns of functional specialization, serving as genetic fingerprints (Stansberg et al., 2007). The transcriptional profiles of the rat frontomedial, temporal and occipital cortices showed several significantly enriched genes in each cortex-sub-region in the right and left

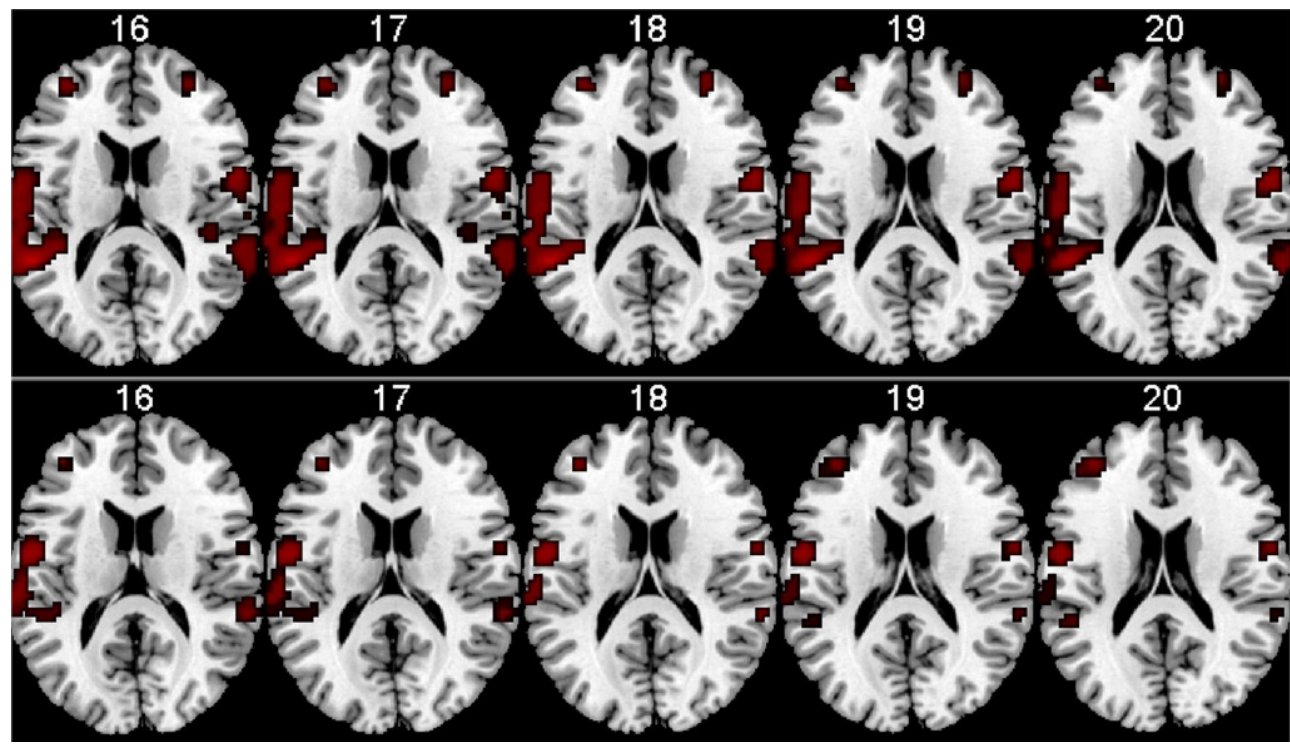

Figure 8. fMRI BOLD activation in the left and right peri-Sylvian areas when listening to dichotic presentations of CV-syllables. The upper row shows activations for the Drug-naive condition, the lower row shows the corresponding activations for the Memantine condition. From left to right: Axial slices corresponding to 16-20 mm above the AC-PC midline in the standardized Talairach and Tournoux (1988) coordinate system. Neurological left-right display conventions. 
hemispheres (Stansberg et al., unpublished data). These genes mainly include transcription factors, ion channels, receptors, and proteases. We are now exploring the existence of a similar specificity of gene expression in human brain postmortem samples with respect to hemisphere asymmetrical expression. We moreover intend to perform case control association studies of these cortex subregion-enriched genes with respect to their potential role in speech perception in general, and in distorted speech perception, as in auditory hallucinations, in particular, based on the hypothesis that auditory hallucinations are related neuronal dysfunction in the inner ear or left peri-Sylvian region.

\section{DISCUSSION AND CONCLUSIONS}

In the present paper, we advance the idea that auditory hallucinations are internally generated speech perceptions that are localized in the left temporal lobe, in the left peri-Sylvian region. If hallucinations are misidentified perceptions originating in the speech perception area of the left temporal lobe, then hallucinating patients should have problems identifying a simultaneously presented external speech sound, especially when the sound is presented lateralized to the left hemisphere. Using a DL paradigm with pair-wise presentations of consonant-vowel syllables we showed that patients with schizophrenia who experience frequent and/or intense auditory hallucinations fail to show an expected REA which may be indicative of a functional deficit in this brain region. The results have also shown that patients who experience frequent hallucinations are impaired in their ability to use top-down cognitive strategies to overcome a bottom-up stimulusdriven right ear effect when instructed to focus attention on the left or right ear stimulus. The behavioral data are supported by MR brain imaging data looking for pathology of grey matter density in patients with schizophrenia, particularly in the left hemisphere. The reviewed studies have revealed significant reductions in grey matter density in the left peri-Sylvian region, including thinning of the cortical mantle, also supported by altered neuronal activation as seen in the fMRI results. Using the drug memantine (ebixa ${ }^{\circledR}$ ) to inhibit the action of glutamate at the NMDA receptor site showed a negative effect on the REA. The fMRI BOLD data moreover showed reduction in neuronal activation in the same regions when subjects were on memantine compared to when the same subjects were drug-naive. Finally, we suggest that deviant gene expression in the left peri-Sylvian region in schizophrenia patients may predispose the patients for auditory hallucinations.

\section{CONFLICT OF INTEREST STATEMENT}

The authors declare that the research was conducted without any commercial or financial relationships that could be construed as a potential conflict of interest.

\section{ACKNOWLEDGEMENTS}

The present work was financially supported by grants from the Research Council of Norway ("Grunnleggende sykdomsmekanismer", "Psykisk helse" and "FUGE" programs), the Health Authorities for Western Norway (Helse Vest RHF) and Dr. Einar Martens fund.

Special thanks to Gesche Neckelmann, Haukeland University Hospital, Norway for analyzing the VBM data.

\section{REFERENCES}

Allen, P., Amaro, E., Fu, C. F., Williams, S. C. R., Brammer, M. J., Johns, L. C., and McGuire, P. K. (2007). Neural correlates of the misattribution of speech in schizophrenia. Br. J. Psychiatry 190, 162-169.

American Psychiatric Association (1994). Diagnostic and Statistical Manual of Mental Disorders (DSM). Washington, D.C.

Ashburner, J., and Friston, K. J. (2001). Why voxel-based morphometry should be used. Neuroimage 14, 1238-1243.

Bartha, R., Al-Semaan, Y. M., Williamson, P. C., Drost, D. J., Malla, A. K., Carr, T. J., Densmore, M., Canaran, G., and Neufeld, W. R. J. (1999). A short echo proton magnetic resonance spectroscopy study of the left mesial-temporal lobe in first-onset schizophrenic patients. Biol. Psychiatry 45, 1403-1411.

Binder, J. R., Frost, J. A., Hammeke, T. A., Rao, S. M., and Cox, R. W. (1996). Function of the left planum temporale in auditory and linguistic processing. Brain 119, 1239-1247.
Binder, J., and Price, C. J. (2001). Functional neuroimaging of language. In Handbook of Functional Neuroimaging of Cognition, A. Kingstone, ed (Cambridge, MA, MIT Press), pp. 187-250.

Blakemore, S., and Frith, C. (2000). Functional neuroimaging studies of schizophrenia. In Brain Mapping: The Disorders, J. C. Mazziotta, A. W. Toga and R. S. J. Frackowiak, eds (San Diego, CA, Academic Press).

Brodal, A. (1981). Neurological anatomy in relation to clinical medicine, $3^{\text {rd }}$ Edn. New York, NY, Oxford University Press.

Bryden, M. P. (1988). An overview of the DL procedure and its relation to cerebral organization. In Handbook of DL: Theory, Methods, and Research, K. Hugdahl ed (Chichester, Wiley and Sons).

Crow, T. J. (1997). Schizophrenia as a failure of the hemispheric dominance for language. Trends Neurosci. 20, 339-343.

Crow, T J. (2004). Auditory hallucinations as primary disorders of syntax: an evolutionary theory of the origins of language. Cogn. Neuropsychiatry 9, 125-145.

David, A. S. (1999). Auditory hallucinations: phenomenology, neuropsychology and neuro-imaging up-date. Acta Psychiatr. Scand. 395, 95-104.

David, A. S. (2004). The cognitive neuropsychiatry of auditory verbal hallucinations: an overview. Cogn. Neuropsychiatry 9, 107-124.

Deary, I. J., and Caryl, P. G. (1997). Neuroscience and human intelligence differences. Trends Neurosci. 20, 365-371.

DeLisi, L. E. (1999). Defining the course of brain structural change and plasticity in schizophrenia. Psychiatry Res. 92, 1-9.

Dierks, T., Linden, D. E. J., Jandl, M., Formisano, E., Goebel, R., Lanfermann, H., and Singer, W. (1999). Activation of Heschl's gyrus during auditory hallucinations. Neuron 22, 615-621.

Espeseth, T., Greenwood, P. M., Reinvang, I., Fjell, A. M., Walhovd, K. B., Westlye, L. T., Wehling, E., Lundervold, A., Rootwelt, H., and Parasuraman, R. (2006). Interactive effects of APOE and CHRNA4 on attention and white matter volume in healthy middle-aged and older adults. Cogn. Affect. Behav. Neurosci. 6, 31-43.

Fan, J., McCandliss, B. D., Fosella, J., Flombaum, J. I., and Posner, M. I. (2005). The activation of attentional networks. Neuroimage 26, 471-479.

Fjell, A. M., Walhovd, K. B., Reinvang, I., Lundervold, A., Salat, D., Quinn, B. T., Fischl, B., and Dale, A. M. (2006). Selecetive increase of cortical thickness in high-performing elederly - structural indices of optimal cognitive aging. Neuroimage 29, 984-994.

Fisher, S. E., Lai, C. S., and Monaco, A. P. (2003). Deciphering the genetic basis of speech and language. Annu. Rev. Neurosci. 26, 57-80.

Frith, C. D. (1992). The Cognitive Neuropsychology of Schizophrenia. Hove, Psychology Press.

Gaser, C., Nenadic, I., Volz, H. P., Büchel, C., and Sauer, H. (2004). Neuroanatomy of 'hearing voices': a frontotemporal brain structural abnormality associated with auditory hallucinations in schizophrenia. Cereb. Cortex 14, 91-96.

Gogtay, N., Giedd, J. N., Lusk, L., Hayashi, K. M., Greenstein, D., Vaituzis, A. K., Nugent, T. F. III, Herman, D. H., Clasen, L. S., Toga, A. W., Rapoport, J. L., and Thompson, P. M. (2004). Dynamic mapping of human cortical development during childhood through early adulthood. Proc. Natl. Acad. Sci. U.S.A. 101, 8179-8174.

Green, M. F., Hugdahl, K., and Mitchell, S. (1994). Dichotic listenig during auditory hallucinations in schizophrenia. Am. J. Psychiatry 151, 357-362.

Heinks-Maldonado, T., Mathalon, D. H., Houde, J. H., Gray, M, Faustman, W. O., and Ford, J. M. (2007). Relationship of imprecise corollary discharge in schizophrenia to auditory hallucinations. Arch. Gen. Psychiatry 64, 286-296.

Hoffman, R. E., Hampson, M., Wu, K., Anderson, A. W., Gore, J. C., Buchanan, R. J., Constable, R. T., Hawkins, K. A., Sahay, N., and Krystal, J. H. (2007). Probing the pathophysiology of auditory/verbal hallucinations by combining functional magnetic resonance imaging and transcranial magnetic stimulation. Cereb. Cortex 17, 2733-2743.

Hoffman, R. E., and McGlashan, T. H. (2006). Using a speech perception neural network computer simulation to contrast neuroanatomic versus neuromodulatory models of auditory hallucinations. Pharmacopsychiatry 39 , Suppl 1, 54-64.

Hubl, D., Koening, T., Strik, W. K., Melie Garcia, L., and Dierks, T. (2007). Competition for neuronal resources: how hallucinations make themselves heard. Br. J. Psychiatry, $190,57-62$

Hugdahl, K. (2003). DL in the study of auditory laterality. In The Asymmetrical Brain, K. Hugdahl and R. J. Davidson, eds (Cambridge, MA, MIT Press), pp. 441-478.

Hugdahl, K., and Andersson, L. (1986). The "forced-attention paradigm" in DL to CVsyllables: a comparison between adults and children. Cortex 22, 417-432.

Hugdahl, K., Brønnick, K., Law, I., Kyllingsbæk, S., and Paulson, O. B. (1999). Brain activation during dichotic presentations of consonant-vowel and musical instruments stimuli: a 150-PET study. Neuropsychologia 37, 431-440.

Hugdahl, K., Løberg, E.-M., Jørgensen, H. A., Lundervold, A., Lund, A., Green, M. F., and Rund, B. R. (2008). Left hemisphere lateralization of auditory hallucinations in schizophrenia: a DL study. Cogn Neuropsychiatry 13, 166-179.

Hugdahl, K., Rund, B. R., Lund, A., Asbjørnsen, A., Egeland, J., Landrø, N. I., Roness, A., Stordal, K., Sundet, K., and Thomsen, T. (2004). Brain activation measured with fMRI during a mental arithmetic task in schizophrenia and major depression. Am. J. Psychiatry 161, 286-293.

Hugdahl, K., Wester, K., and Asbjørnsen, A. (1990). The role of the left and right thalamus in language asymmetry: DL in Parkinson-patients undergoing stereotactic thalamotomy. Brain Lang. 39, 1-13. 
Kay, S. R., Opler, L. A., and Fiszbein, A. (1989). SCI-PANSS. North Tonawanda, NY, MultiHealth Systems Inc.

Kimura, D. (1961). Cerebral dominance and the perception of verbal stimuli. Can. J. Psychol. 15, 166-171.

Kimura, D. (1967). Functional asymmetry of the brain in DL. Cortex 3, 163-168.

Kinderman, P. (2007). Auditory hallucinations. J Norw Psychol Assoc 44, 547-554.

Kubicki, M., Shenton, M. E., Salisbury, D. F., Hirayasu, Y., Kasai, K., Kikinis, R., Jolesz, F. A., and McCarley, R. W. (2002). Voxel-based morphometric analysis of gray matter in first episode schizophrenia. Neuroimage 17, 1711-1719.

Lai, C. S., Fisher, S. E., Hurst, J. A., Vargha-Khadem, F., and Monaco, A. P. (2001). A forkhead-domain gene is mutated in a severe speech and language disorder. Nature 413, 519-523.

Lawrie, S. M., and Abukmeil, S. S. (1998). Brain abnormality in schizophrenia. A systematic and quantitative review of volumetric magnetic resonance imaging studies Br. J. Psychiatry 172, 110-120.

Liegeois, F., Baldeweg, T., Connelly, A., Gadian, D. G., Mishkin, M., and Vargha-Khadem, F. (2003). Language fMRI abnormalities associated with FOXP2 gene mutation. Nat Neurosci. 6, 1230-1237.

Løberg, E. M., Hugdahl, K., and Green, M. F. (1999). Hemispheric asymmetry in schizophrenia: a "dual deficits" model. Biol. Psychiatry 45, 76-81.

Løberg, E. M., Jørgensen, H. A., and Hugdahl, K. (2004). DL in schizophrenic patients: effects of previous vs. ongoing auditory hallucinations. Psychiatry Res. 128, 167-174.

Lukoff, D., Nuechterlein, K. H., and Ventura, J. (1986). Appendix A: manual for the expanded brief psychiatric rating scale (BPRS). Symptom monitoring in the rehabilitation of schizophrenic patients. Schizophr. Bull. 12, 594-602.

Neckelmann, G. Specht, K., Lund, A., Ersland, L., Smievoll, Al., and Hugdahl, K. (2006) MR morphometry analaysis of grey matter density reducution in schizophrenia: interactions with hallucinations. Int. J. Neurosci. 116, 9-23.

Plaze, M., Bartrés-Faz, D., Martinot, J. L., Januel, D., Bellivier, F., De Beaurepaire, R. Chanraud, S., Andoh, J., Lefaucheur, J.-P., Artiges, E., Pallier, C., and PaillèreMartinot, M.-L. (2006). Left superior temporal gyrus activation during sentence perception negatively correlates with auditory hallucination severity in schizophrenia patients. Schizophr. Res. 87, 109-115.

Pollmann, S., Lepsien, J., Hugdahl, K., and von Cramon, D. Y. (2004). Auditory target detection in $\mathrm{DL}$ involves the orbitofrontal and hippocampal paralimbic belts. Cereb. Cortex 14, 903-913.

Posner, M. I., and Raichle, M. E. (1994). Images of Mind. New York, NY, W. H. Freeman and $\mathrm{Co}$.

Posner, M. I. and Rothbart, M. (2007). Research on attention networks as a model for the integration of psychological science. Annu. Rev. Neurosci. 58, 1-23.

Salat, D. H., Buckner, R. L., Snyder, A. Z., Greve, D. N., Desikan, R. S. R., Busa, E., Morris, J. C., Dale, A. M., and Fischl, B. (2004). Thinning of the cerebral cortex in aging. Cereb. Cortex 14, 721-730.

Sanjuan, J., Toirac, I., Gonzalez, J. C., Leal, C., Molto, M. D., Najera, C., and de Frutos R. (2004). A possible association between the CCK-AR gene and persistent auditory hallucinations in schizophrenia. Eur. Psychiatry 19, 349-353.

Sanjuan, J., Tolosa, A., Gonzalez, J. C., Aguilar, E. J., Perez-Tur, J., Najera, C., Molto M. D., and de Frutos, R. (2006). Association between FOXP2 polymorphisms and schizophrenia with auditory hallucinations. Psychiatr. Genet. 16, 67-72.

Shergill, S. S., Brammer, M. J., Amaro, E., Williams, S. C. R., Murray, R. M., and McGuire, P. K (2004). Temporal course of auditory hallucinations. Br. J. Psychiatry 185, 516-517.

Shergill, S. S., Brammer, M. J., Williams, S. C. R., Murray, R. M., and McGuire, P. K (2000). Mapping auditory hallucinations in schizophrenia using functional magnetic resonance imaging. Arch. Gen. Psychiatry 57, 1033-1038.
Siegel, C., Waldo, M., Gizner, G., and Adler, L. E. (1984). Deficits in sensory gating in schizophrenic patients and their relatives. Evidence obtained with auditory evoked responses. Arch. Gen. Psychiatry 41, 607-612.

Silbersweig, D. A., Stern, E., Frith, C., Cahill, C., Holmes, A., Grootonk, S., Seaward, J., McKenna, P., Chua, S. E., Schnorr, L., Jones, T., and Frackowiak, R. J. S. (1995). A functional neuroanatomy of hallucinations in schizophrenia. Nature 378 , 176-179.

Sperry, R. W. (1974). Lateral specialization in the surgically separated hemispheres. In The Neuroscience: Third Study Program, F. O. Schmitt and F. G. Worden, eds (Cambridge, MA, MIT Press), pp. 5-19.

Spitzer, R. L., Williams, J. B. W., Gibbon, M., and First, M. B. (1990). User's Guide for the Structured Clinical Interview for the DSM-III-R (SCID). Washington, DC, American Psychiatric Press.

Stansberg C., Vik-Mo, A. O., Holdhus, R., Breilid, H., Srebro, B., Petersen, K., Jørgensen, H. A., Jonassen, I., and Steen, V. M. (2007). Gene expression profiles in rat brain disclose CNS signature genes and regional patterns of functional specialisation. BMC Genomics 8, 94

Sun, T., Patoine, C., Abu-Khalil, A., Visvader, J., Sum, E., Cherry, T. J., Orkin, S. H., Geschwind, D. H., and Walsh, C. H. (2005). Early asymmetry of gene transcription in embryonic human left and right cerebral cortex. Science 308, 1794-1798.

Talairach, J., and Tournoux, P. (1988). Co-Planar Stereotaxic Atlas of the Human Brain 3-D Propotional System: An Approach to Cerebral Imaging. New York, NY, Georg Thieme Verlag.

Tervaniemi, M., and Hugdahl, K. (2003). Lateralization of auditory-cortex functions. Brain Res. Rev. 43, 231-246.

Toirac, I., Sanjuan, J., Aguilar, E. J., Gonzalez, J. C., Artigas, F., Rivero, 0., Najera, C., Molto, M. D., and de Frutos, R. (2007). Association between CCK-AR gene and schizophrenia with auditory hallucinations. Psychiatr. Genet. 17, 47-53.

Tritsch, N. X., Eunyoung, Y. I., Gale, J. E., Glowatzki, E., and Bergles, D. E. (2007). The origin of spontaneous activity in the developing auditory system. Nature 450, doi: 10.1038, Epub ahead of publication

van den Noort, M., Specht, K., Rimol, L. M., Ersland, L., and Hugdahl, K. (2007). A new verbal reports fMRI dichotic listening paradigm for studies of hemispheric asymmetry. Neuroimage (epub ahead of print PMID: 18234509).

van der Gaag, M. (2006). A neuropsychiatric model of biological and psychological processes in the remission of delusions and auditory hallucinations. Schizophr. Bull. $32,113-122$.

Vargha-Khadem, F., Watkins, K., Alcock, K., Fletcher, P., and Passingham, R. (1995). Praxic and nonverbal cognitive deficits in a large family with a genetically transmitted speech and language disorder. Proc. Natl. Acad. Sci. U.S.A. 92, 930-933.

Wei, J., and Hemmings, G. P. (1999). The CCK-A receptor gene possibly associated with auditory hallucinations in schizophrenia. Eur. Psychiatry 14, 67-70.

Wing, J. K., Cooper, J. E., and sartorius, N. (1974). The Measurement and Classification of Psychiatric Symptoms: An Instructional Manual for teh PSE and CATEGO Programs. New York, NY, Cambridge University Press.

Woodruff, P. W. R. (2004). Auditory hallucinations: insights and questions from neuroimaging. Cogn. Neuropsychiatry 9, 73-92.

Woodruff, P. W. R., Wright, I., Bullmore, E., Brammer, M., Howard, R. J., Williams, S. C. R., Shapleske, J., Rossell, S., David, A., McGuire, P., and Murray, R. M. (1997) Auditory hallucinations and the temporal cortical response to speech in schizophrenia: a functional magnetic resonance imaging study. Am. J. Psychiatry 154, 1676-1682.

World Health Organization (1992).The ICD-10 classification of mental and behavioural disorders: Clinical descriptions and diagnostic guidelines. Geneva, Switzerland. 